\title{
Outcome of In Vitro Fertilization in Women with Discordant Values of Anti Mullerian Hormone and Antral Follicle Count
}

\author{
Swati Verma ${ }^{1 *}$, Umesh Nandini Jindal ${ }^{2}$, Sonia Gupta ${ }^{3}$, Sanjeev Maheshwari ${ }^{4}$ and Bharti Joshi ${ }^{5}$ \\ ${ }^{1}$ Senior consultant, Department of reproductive medicine, Jindal IVF and Sant Memorial Nursing Home, India \\ ${ }^{2}$ Director, Department of reproductive medicine, Jindal IVF and Sant Memorial Nursing, India \\ ${ }^{3}$ Ex resident of Department of reproductive medicine, Jindal IVF and Sant Memorial Nursing Home, India \\ ${ }^{4}$ Senior embryologist, Department of reproductive medicine, Jindal IVF and Sant Memorial Nursing Home, India \\ ${ }^{5}$ Ex-assistant Professor, Department of Obstetrics and Gynaecology, Post Graduate Institute of Medical Education and Research, India
}

Submission: April 10, 2018; Published: May 31, 2018

*Corresponding author: Dr Swati Verma, Senior consultant, Jindal IVF center, sector 20 D, Chandigarh, India, Tel: 9646004459; Email: swati7562@yahoo.com

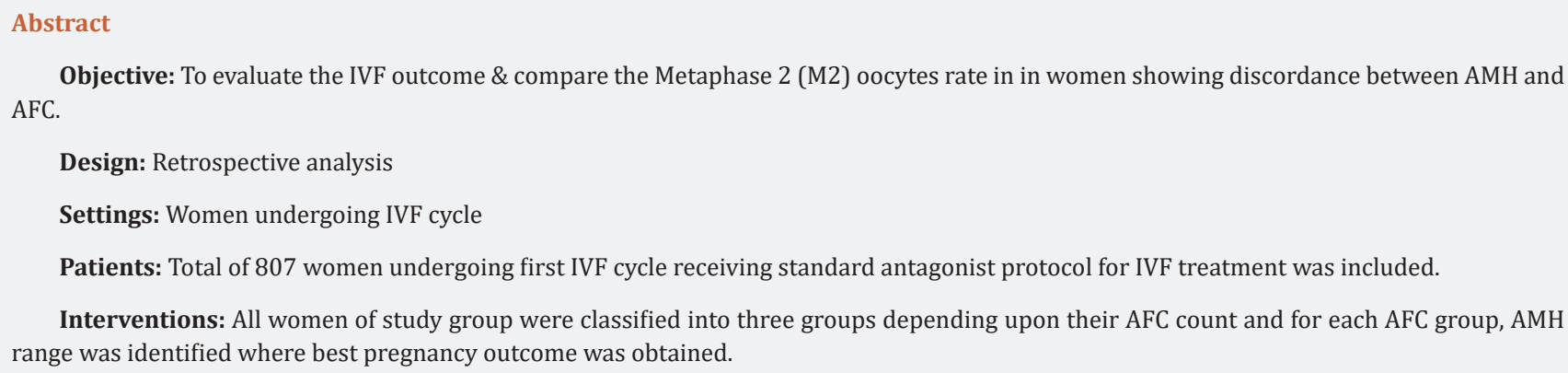

Main Outcome measures: IVF outcome in women with discordant AFC \& AMH.

Results: For each AFC group, AMH cut off values were different according to their respective percentiles. Only 20.07\% ( 162/807) women among three AFC groups were observed showing concordance between AMH and AFC. Within each AFC group women showing concordance between AMH and AFC were associated with better IVF outcomes. Despite higher M2 rates reported with higher AMH values in each AFC category, lower pregnancy rates were reported.

Conclusion: Discordance between AFC and AMH they may have adverse impact on outcome of IVF.

Keywords: Concordant; Discordant; Antral follicle Count; Anti-Mullerian hormone; In vitro fertilization; Ovarian Reserve; Retrieved oocytes; Metaphase 2; Serum Follicle; Embryo transfer; Transvaginal Ultrasound; ELISA kit; Gonadotrophins; Oestradiol Levels; Aetiology of infertility; Granulosa Cells; Antral follicles; Pregnancy rate; Chi Square Test; Luteal Phase; Follicular monitoring

Abbreviations: AMH: Anti-Mullerian Hormone; IVF: In Vitro Fertilization; AFC: Antral Follicle Count; S.FSH: Serum Follicle Stimulating Hormone; PCO: Polycystic Ovaries; M2: Metaphase 2

\section{Introduction}

Among various proposed biomarkers, Antral Follicle Count (AFC) and Anti Mullerian Hormone (AMH) are said to be most favorable in predicting ovarian reserve and response to stimulation.Both generally have good correlation with each other and are often used interchangeable. There is a growing evidence of consistent association of these biomarkers with ovarian response, number of retrieved oocytes and live birth rate [1-4]. Nonetheless discordance between the two does occur and has been discussed in recent trials [5,6]. The discordance between $\mathrm{AFC}$ and $\mathrm{AMH}$ raises a doubt regarding the usefulness of $\mathrm{AMH}$ 
or possibility of some laboratory error [7]. It may also suggest existence of some subtle ovarian pathology, not demonstrated by ultrasonography done for assessment of AFC. A very limited data exits on IVF outcome in women with discordance between AMH and AFC. Therefore we conducted index review among women undergoing In-Vitro Fertilization (IVF) at our center with the following objectives;

a) To define the discordance between $\mathrm{AMH}$ and $\mathrm{AFC}$ in women undergoing IVF

b) To study the IVF outcome in women showing discordance between $\mathrm{AMH}$ and $\mathrm{AFC}$

c) To compare the Metaphase 2 (M2) oocytes rate in women with discordant AMH and AFC in relation to their IVF outcome

\section{Study Design, Materials \& Methods}

This retrospective analysis was conducted from January 2012 to December 2015 at an infertility centre of North India. There were 1754 women who underwent IVF cycle during this period. After fulfilling inclusion criteria 807 women were found eligible for the study (Figure 1). The women included in the analysis were with their age below 35 years, day two serum follicle stimulating hormone (S.FSH) levels below $10 \mathrm{IU} / \mathrm{ml}$, first IVF cycle with self oocytes, received ovarian stimulation with standard GnRH antagonist protocol along with fresh embryo transfer done. Most of the women with polycystic ovaries (PCO) were excluded from the study as fresh embryo transfer was not possible to avoid risk of OHSS. The women with PCO who underwent fresh ET were included in the analysis. Although this was a retrospective analysis and did not involve any active intervention on patients, approval was taken from the institutional review board.

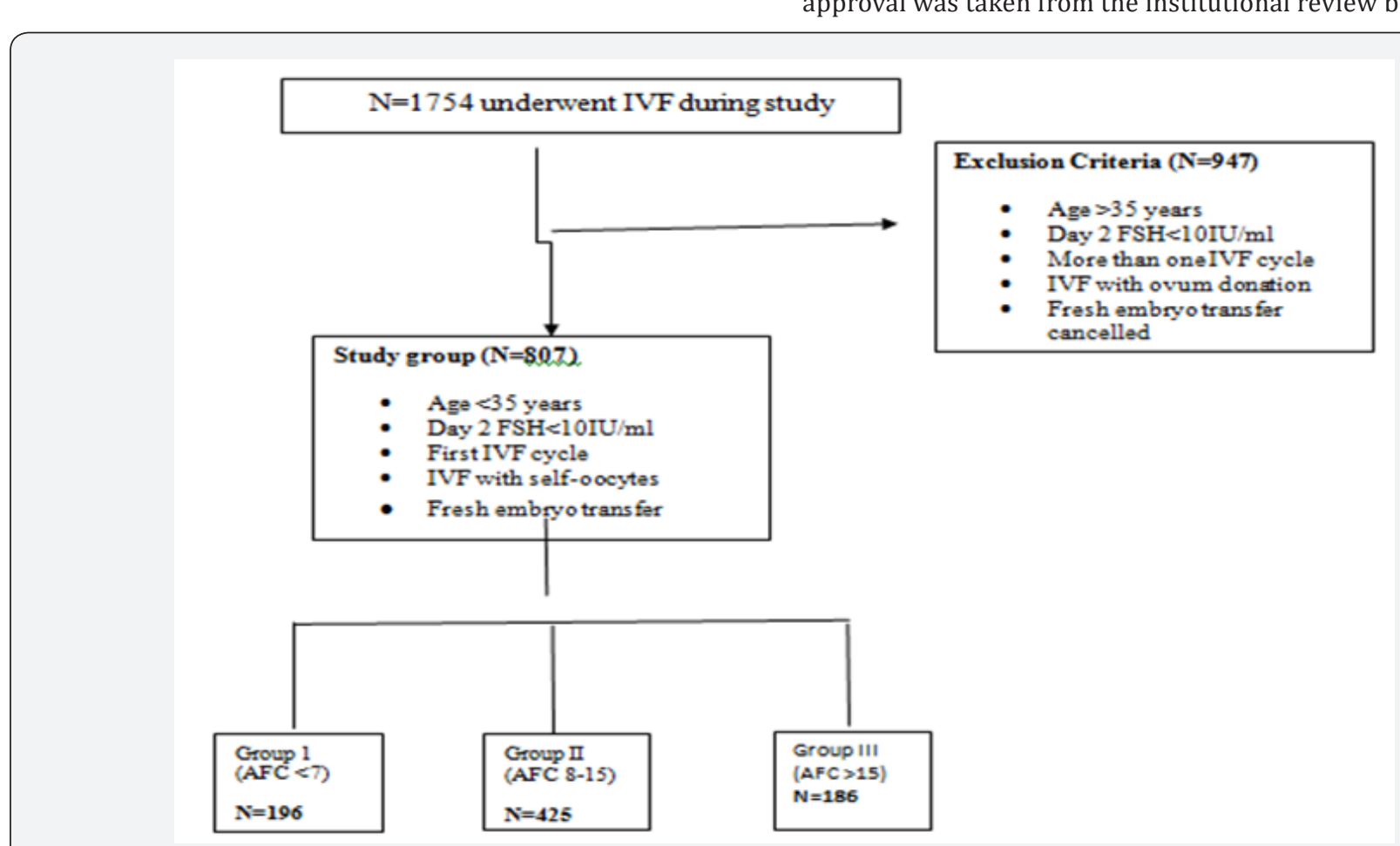

Figure 1: Inclusion and exclusion criteria of study group.

\section{AFC and AMH determination}

The ovarian reserve was assessed by AFC, serum AMH and day 2 S.FSH as a routine. Total number of AFC was measured on day 2 of cycle by transvaginal ultrasound scan and all the follicles ranging from 2 to $9 \mathrm{~mm}$ were included. AMH measurement was done using the AMH Gen II ELISA kit. The assay kit has a sensitivity of $0.08 \mathrm{ng} / \mathrm{ml}$, and intra- and inter-assay coefficients of variation of less than 5.4 and 5.6 respectively.

\section{Ovarian stimulation protocol}

Standard GnRH antagonist protocol for ovarian stimulation was followed in all women. Starting dose of injection Gonadotrophins was decided according to the value of AFC, AMH and S. FSH. Ultrasound guided follicular monitoring was done and injection $\mathrm{GnRH}$ antagonist. $25 \mathrm{mg} \mathrm{S} / \mathrm{C}$ was added once one or more follicles reaches $13-14 \mathrm{~mm}$ in size. Dosages of injection Gonadotrophins were adjusted according to ovarian response. Injection urinary hCG 10000 I.U was given as oocyte maturation trigger in the patients who were not at risk of OHSS. Women with more than 12 follicles of $16 \mathrm{~mm}$ and serum oestradiol levels more than $3500 \mathrm{pg} / \mathrm{ml}$ were given modified oocytes maturation trigger with injection decapeptyl $0.2 \mathrm{mg}$ subcutaneous. Oocyte retrieval was done 34-36 hours after the maturation trigger. All women received injection progesterone $50 \mathrm{mg}$ intramuscular daily for luteal phase support, started after oocyte retrieval. Women who received modified maturation trigger were administered 


\section{Global Journal of Reproductive Medicine}

injection urinary hCG 1500I.U on the day of oocyte retrieval and tablet oestradiol valerate $2 \mathrm{mg}$, 8 hourly along with standard luteal phase support. All women were subjected to embryo transfer with two good quality embryos on day 2 of oocyte retrieval.

\section{Distribution of subjects and finding cut-off values for AMH in each AFC group}

Study group were categorized into three groups according to AFC. Group 1: $\leq 7$ (n=196), Group II: 8-15 ( $\mathrm{n}=425)$, Group III: >15 $(n=186)$. For each AFC group ( $\leq 7,8-15$ and $>15)$, entire range of AMH values were binned at 5 percentile values using SPSS 16. Total 20 intervals of AMH values over whole range of data were Results obtained. IVF outcome (pregnancy yes and no) over various AMH intervals was plotted as histogram. An interpolation line was drawn to demonstrate the trend using two moving average of Excel. Change of trend in IVF outcomes was noted by observing the graph and corresponding cut off values for AMH were defined.

\section{Statistical analysis}

According to the cut off AMH values three subgroups i.e. low discordant, concordant and high discordant were obtained within each AFC group. IVF outcomes in each of three groups were analyzed comparing three sub groups using Pearson Chi Square test and the level of significance was calculated. The twotailed value of $\mathrm{P}<0.05$ was considered statistically significant.

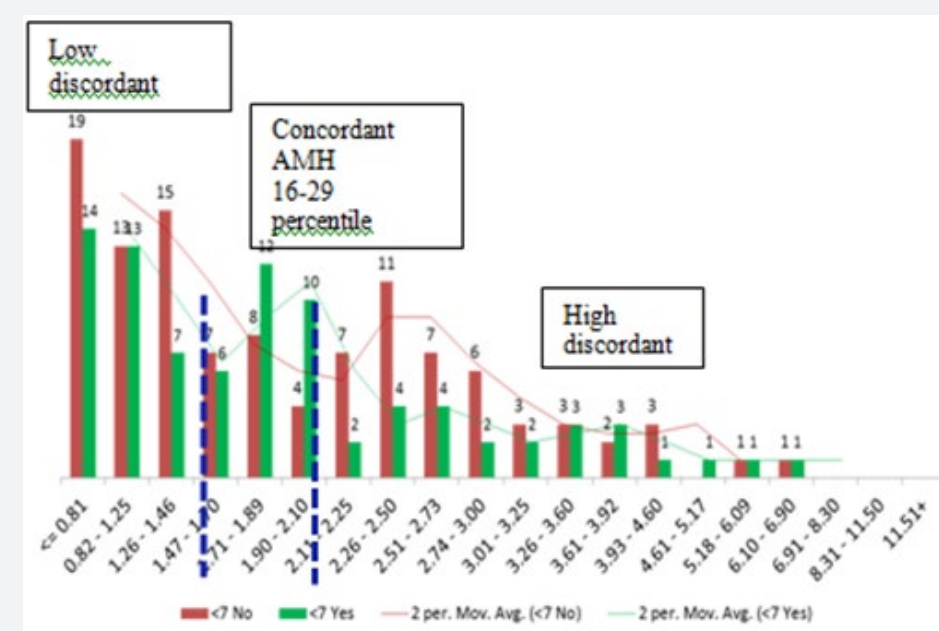

Figure 2: AMH cut off range for Group I.

Group $1 \mathrm{AFC}=<7$.

Figure 3: AMH cut off range for Group II.

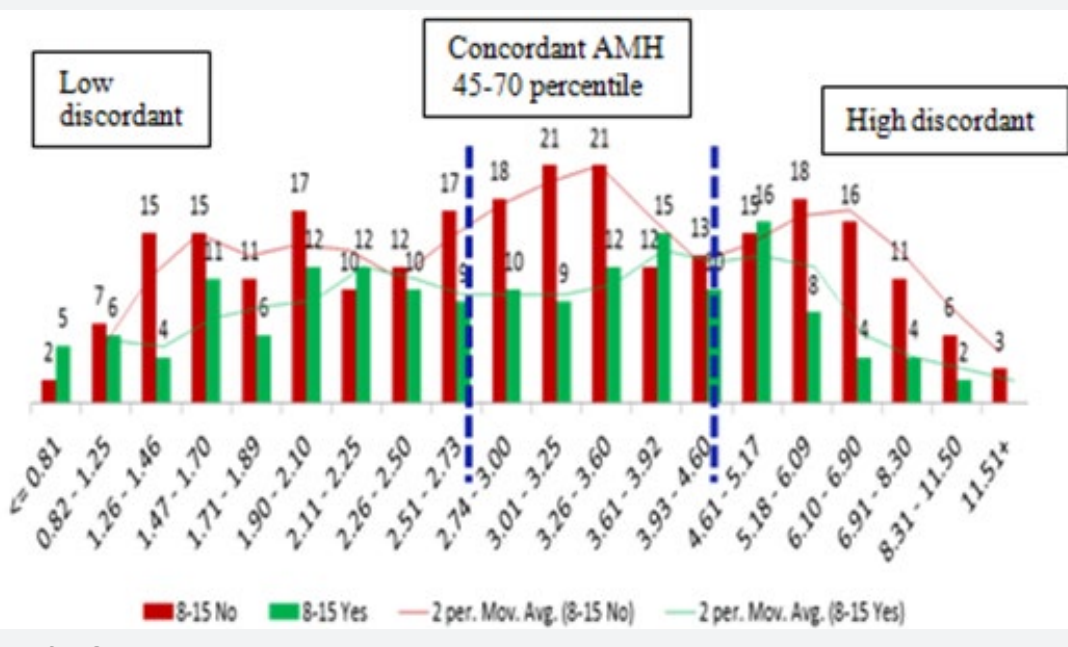

Group II AFC: 8-15.

For each AFC group, AMH cut off values were different according to their respective percentiles (Figure 2-4). Only $20.07 \%$ (162/807) women among three AFC groups were observed showing concordance between AMH and AFC. One third of women i.e. 33.7\% (272/807) included in the study had higher AMH values (high discordance) whereas 46.22\% (373) had 


\section{Global Journal of Reproductive Medicine}

lower AMH values (low discordance) than expected according to their AFC (Table 1). The three subgroups within each AFC group were comparable for age, duration and aetiology of infertility and indications for IVF. Within each AFC group women showing concordance between AMH and AFC were associated with better IVF outcomes (Table 2). Pregnancies in women in Group 1 and Group III with both high and low discordant AMH values were comparable (42\% Vs $43.9 \%$ and $30 \%$ Vs $32.6 \%$ ). Women with AFC 8-15 but either high AMH i.e. Group II with high discordance had lowest pregnancy rate $(25 \%)$. Although two other AFC Groups i.e.1 and III with high discordant AMH values did not follow the same trend, pregnancy rate was $43.9 \%$ and $32.6 \%$ and it was higher compared with their respective low AMH subgroup women (Figure 2-3). Highest PR was observes in women of Group 1 with concordance AMH values (59.6\%). Women with low discordant $\mathrm{AMH}$ values of all three AFC groups were reported with lower (Group I, II and III; 42\%, 39\% and 30\% respectively). Women in Group III with high discordance (this group mainly consisted of PCOS women) had PR 32.6\%; indicating that higher AFC with higher AMH also did not have higher conception rate when embryos were transferred in the same cycle.

Table 1: AMH cut off values in different AFC groups.

\begin{tabular}{|c|c|c|c|}
\hline Subgroup & Group 1: $\leq 7$ & Group II:8-15 & Group II:>15 \\
\hline & AMH cut off $\mathrm{ng} / \mathrm{ml}(\mathrm{n})$, percentile & AMH cut off $\mathrm{ng} / \mathrm{ml}(\mathrm{n})$, percentile & AMH cut off $n g / m l(n)$, percentile \\
\hline Low discordant & $<1.48 \mathrm{ng} / \mathrm{ml}(\mathrm{n}=81)$ & $<3.61(\mathrm{n}=272)$ & $<2.74(\mathrm{n}=20)$ \\
\hline $46.22 \%(373)$ & $<16$ percentile & (<60percentile) & $(<45$ percentile $)$ \\
\hline Concordant & $1.48-2.10(n=47)$ & $3.6-5.17(n=81)$ & $2.74-4.60(n=34)$ \\
\hline $20.07 \%(162)$ & (16-29 percentile) & (60-75 percentile) & (45-70percentile) \\
\hline High discordant & $>2.10(68)$ & $>5.17(\mathrm{n}=72)$ & $>4.6(\mathrm{n}=132)$ \\
\hline $33.71 \%(272)$ & (> 29 percentile) & (> 75 percentile) & (> 70 percentile) \\
\hline Total $=\mathbf{8 0 7}$ & $196(24.28 \%)$ & $425(52.66 \%)$ & $186(23.06 \%)$ \\
\hline
\end{tabular}

Table 2: Pregnancy outcome in different AFC groups.

\begin{tabular}{|c|c|c|c|c|c|c|}
\hline \multirow{2}{*}{ Subgroup } & \multicolumn{2}{|c|}{ Group 1: $\leq 7$ Pregnancy } & \multicolumn{2}{|c|}{ Group II: 8-15 Pregnancy } & \multicolumn{2}{|c|}{ Group II:>15 Pregnancy } \\
\hline & No & Yes & No & Yes & No & Yes \\
\hline \multirow{2}{*}{ Low discordant } & 47 & 34 & 166 & 106 & 14 & 6 \\
\hline & $(58 \%)$ & $(42 \%)$ & $(61 \%)$ & $(39 \%)$ & $(70 \%)$ & $(30 \%)$ \\
\hline \multirow{2}{*}{ Concordant } & 19 & 28 & 40 & 41 & 15 & 19 \\
\hline & $(40 \%)$ & $(59.60 \%)$ & $(49.4 \%)$ & $(50.60 \%)$ & $(44.1 \%)$ & $(55.90 \%)$ \\
\hline \multirow{2}{*}{ High discordant } & 110 & 86 & 54 & 18 & 89 & 43 \\
\hline & $(56.10 \%)$ & $(43.9 \%)$ & $(75 \%)$ & $(25 \%)$ & $(67.4 \%)$ & $(32.6 \%)$ \\
\hline$P$ value & \multicolumn{2}{|c|}{.03} & \multicolumn{2}{|c|}{.005} & \multicolumn{2}{|c|}{.034} \\
\hline
\end{tabular}

Mean M2 oocytes rate for pregnancy positive versus negative women among three groups is shown in Figure $5 \& 6$. In the women with concordant AMH values mean M2 oocytes rate for IVF outcome positive vs. negative were in Group I, II and III were 4.33 Vs 2.98; p value.028, 8.8 vs. 6.8 p value .032 and 10.11 vs 9.06; $p$ value .048 respectively. The women of all subgroups with positive IVF outcome demonstrated higher number of M2 rate compared to negative IVF outcome and difference was also statistically significant. The number of M2 oocytes also showed rising pattern corresponding to the rise with $\mathrm{AMH}$ values within each AFC category.

\section{Discussion}

$\mathrm{AMH}$ has emerged as reliable indicator and autonomous marker of ovarian function because of its strong correlation with AFC and operator independency. Earlier there were various controversies regarding $\mathrm{AMH}$ values in relation to laboratory assays. But now a well-established assay Gen II EUSA is available to measure $\mathrm{AMH}$; as standard techniques worldwide. Comparable performance of AMH and AFC in IVF treatment has been documented and may be explained to certain extent by the fact that source of AMH is granulosa cells of antral follicles $[8,9]$. Various studies have described discordance between AMH and S.FSH in women undergoing assisted reproduction. But so far, to the best of our knowledge, there is hardly any literature describing discordance among $\mathrm{AMH}$ and $\mathrm{AFC}$ and their clinical implications .Our study is first of its kind to demonstrate the concordant AMH values according to outcome of IVF. We can not imply the same AMH cut off values to all the women with different AFC. Therefore it is relevant to find out the individual cut off values for different AFC groups, thereafter to correlate the ovarian response and IVF outcome. 


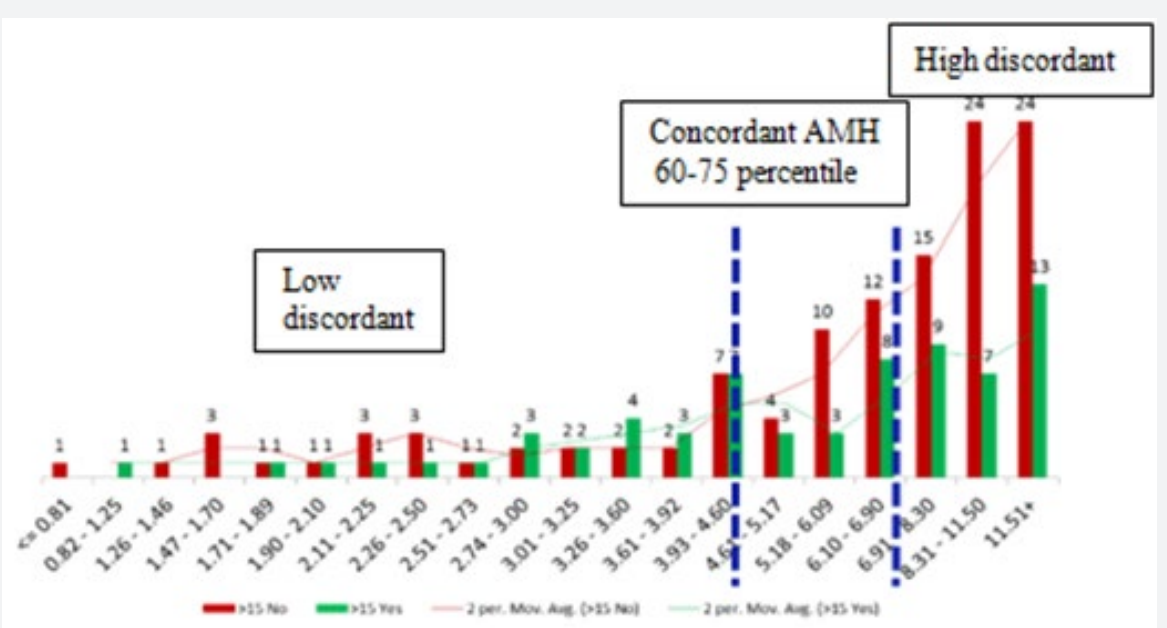

Figure 4: AMH cut off range for Group III.

Group III AFC $>15$.
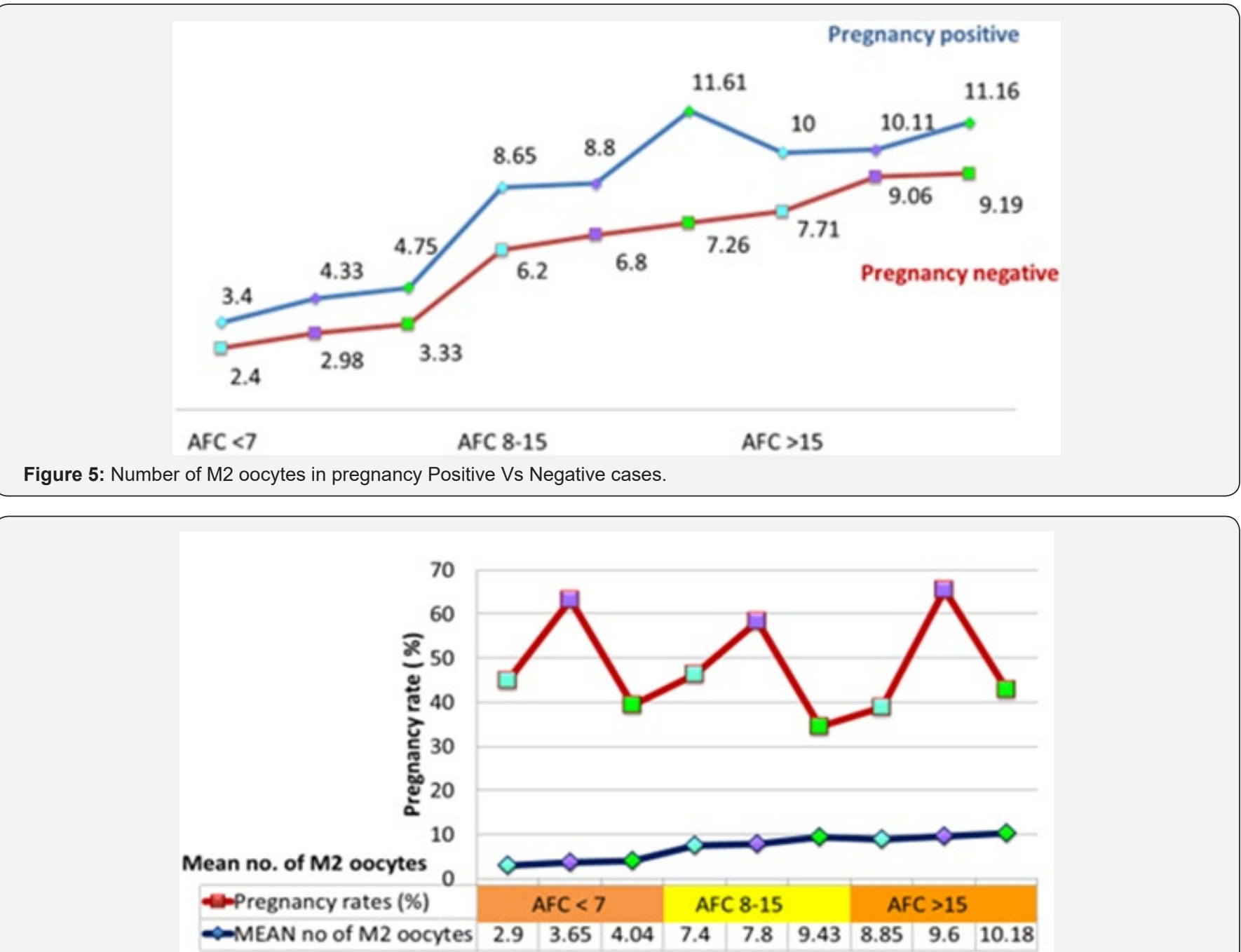

Figure 6: Correlation of M2 oocytes number with pregnancy rates.

Index study showed a significant proportion, amounting to $79.93 \%$, of women undergoing IVF treatment at the centre were showing discordant between $\mathrm{AMH}$ and AFC as classified by their percentiles according to their IVF outcome .There was wide fluctuation of percentile values of concordance of AHM for each AFC group, which indicate that same AHM value 
for different AFC group women may not generate same result. Although there was linear correlation of number of M2 oocytes and good quality embryos with AMH values but IVF outcome did not follow the same trend. When IVF outcome (pregnancy yes and no) was plotted over various AMH intervals as histogram, we found highest pregnancy rate in all concordant groups. Low and high AMH values in all AFC categories were associated with poorer pregnancy rate. For better IVF outcome, there should be a correlation between AFC and AMH. Though this is retrospective data analysis, strength lies in the fact that discordant value of $\mathrm{AMH}$ for every AFC group was described. This is in contrast to study by Raymond Li et al. [10] in which the same AMH range was generalised for the whole study group [10].

\section{Conclusion}

Current study described AMH range for each AFC category where best IVF results were obtained for our centre. Highest pregnancy rates were observed in all three AFC categories showing concordance between AFC and AMH values. Low and high $\mathrm{AMH}$ values in all AFC categories were associated with poorer pregnancy rate. M2 rate increased linearly with rise of $\mathrm{AMH}$ values in each category. Despite higher M2 rates reported with higher AMH values in each AFC category, lower pregnancy rates were reported. Women with negative pregnancy results showed significantly low numbers of M2 oocytes when compared with positive pregnancy within each AFC and AMH category. We hypothesised that discordance between the two may have adverse impact on outcome of IVF. Accumulation of more data is needed to further validate our study.

\section{References}

1. Broer SL, Dólleman M, Opmeer BC, Fauser BC, Mol BW, et al. (2011) $\mathrm{AMH}$ and $\mathrm{AFC}$ as predictors of excessive response in controlled ovarian hyperstimulation: a meta-analysis. Hum Reprod Update 17(1): 46-54.
2. Broer SL, Dólleman M, van Disseldorp J, Broeze KA, Opmeer BC, et al. (2013) Prediction of an excessive response in in-vitro fertilization from patient characteristics and ovarian reserve tests and comparison in subgroups: an individual patient data meta-analysis. Fertil Steril 100(2): 420-429.

3. Iliodromiti S, Kelsey TW, Wu O, Anderson RA, Nelson SM (2014) The predictive accuracy of anti-Mullerian hormone for live birth after assisted conception: a systematic review and meta-analysis of the literature. Hum Reprod Update 20(4): 560-570.

4. La Marca A, Papaleo E, Grisendi V, Argento C, Giulini S, et al. (2012) Development of a nomogram based on markers of ovarian reserve for the individualisation of the follicle-stimulating hormone starting dose in in-vitro fertilisation cycles. BJOG 119(10): 1171-1179.

5. Arce JC, la Marca A, Klein BM, Nyboe Andersen A, Fleming R (2013) Reply of the authors. Fertil Steril 100(2): e10.

6. Andersen AN, Witjes H, Gordon K, Mannaerts B (2011) Predictive factors of ovarian response and clinical outcome after IVF/ICSI following a $\mathrm{rFSH} / \mathrm{GnRH}$ antagonist protocol with or without oral contraceptive pre-treatment. Hum Reprod 26(12): 3413-3423.

7. Broer SL, Mol B, Dolleman M, Fauser BC, Broekmans FJ (2010) The role of anti-Mullerian hormone assessment in assisted reproductive technology outcome. Curr Opin Obstet Gynecol 22(3): 193-201.

8. Broer SL, Mol BWJ, Hendriks D, Broekmans FJM (2009) The role of antimullerian hormone in prediction of outcome after IVF: comparison with the antral follicle count. Fertil Steril 91(3): 705-714.

9. Broer SL, Dolleman M, Opmeer BC, Fauser BC, Mol BW, et al. (2011) $\mathrm{AMH}$ and $\mathrm{AFC}$ as predictors of excessive response in controlled ovarian hyperstimulation: a meta-analysis. Hum Reprod Update 17(1): 46-54.

10. Li HWR, Lee VCY, Lau EYL, Yeung WSB, Ho PC, et al. (2013) Role of baseline antral follicle count and anti-mullerian hormone in prediction of cumulative live birth in the first in-vitro fertilisation cycle: a Retrospective cohort analysis. PLoS One 8(4): e61095.

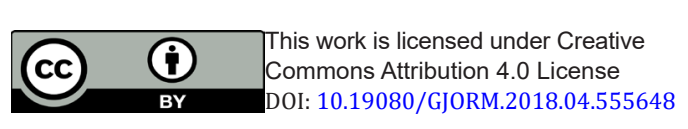

Your next submission with Juniper Publishers
will reach you the below assets
- Quality Editorial service
- Swift Peer Review
- Reprints availability
- E-prints Service
- Manuscript Podcast for convenient understanding
- Global attainment for your research
- Manuscript accessibility in different formats
( Pdf, E-pub, Full Text, Audio)
- Unceasing customer service
Track the below URL for one-step submission
https://juniperpublishers.com/online-submission.php

